\title{
Die Berechnung der totalen Menge gelöster Stoffe in Seen
}

Von H. Bührer

EAWAG, Abteilung Limnologie, Dübendorf

Manuskript eingegangen am 24.August 1979

\section{ABSTRACT}

Formula for estimation of the total content of a substance in lakes

Total content is used mostly for balances. In order to calculate the areas of different depths, the contours are cut out of a topographical map and weighed.

The formula is derived from the substance concentration (taken as a linear function of the depth), times the volume of a truncated cone.

Content of one layer:

$$
\frac{\Delta z}{12}\left[\left(f_{1}+f_{u}\right)^{2} \cdot\left(c_{1}+c_{u}\right)+2 c_{1} f_{1}^{2}+2 c_{u} f_{u l}^{2}\right.
$$

$f_{u} \quad$ Square root of upper area

$f_{1}$ Square root of lower area

$c_{\mathbf{u}}$ Concentration at upper depth

$c_{1}$ Concentration at lower depth

$\Delta \mathrm{z}$ Difference of depths (i.e. thickness of layer)

Sum of contents of all layers gives total content of the lake.

Bei der Bearbeitung mancher limnologischer Fragen und Seeüberwachungen interessieren nicht nur die aktuellen Werte der chemischen Parameter im vertikalen Verlauf, sondern (namentlich bei Bilanzfragen) die Gesamtmenge der betreffenden Stoffe im See. Für die Berechnung dieser Summenwerte stehen normalerweise Einzeldaten zur Verfügung, welche aus den punktförmig aus verschiedenen Tiefen erhobenen Proben ermittelt wurden. Messungen, die das ganze Vertikalprofil kontinuierlich erfassen, sind kaum jemals erhältlich. Eine Formel zur Berechnung des Stoffinhalts des Sees muss dieser Tatsache angepasst sein.

\section{Topographie des Sees}

Für die Berechnung des Volumens werden die Flächen möglichst vieler Tiefen benötigt. Diese Flächen sind in den topographischen Karten durch die Höhenkurven eingezeichnet und können durch Planimetrie erhalten werden. Dieses Verfahren erwies sich aber als ebenso mühsam wie ungenau. Erheblich sicherer ist 
es, die Tiefenflächen mit der Schere auszuschneiden und die dadurch entstandenen Flächendifferenzen auf der Analysenwaage zu wägen. Die gleichmässige Papierqualität der «Schweiz. Landeskarte» erlaubt ein solches Verfahren ohne weiteres. Dass dafür mindestens ein Kartenblatt geopfert werden muss, ist nicht zu vermeiden.

Die so gewonnenen Flächen, welche mit Hilfe einer Eichfläche (ebenfalls aus der topographischen Karte ausgeschnitten) berechnet werden, werden als ebene Flächen verwendet; auf eine Korrektur auf Meereshöhe sowie die Korrektur der Erdkrümmung wird verzichtet. Die Abweichungen durch die Kartenprojektion werden ebenfalls nicht berücksichtigt. Diese Korrekturen wären geringer als die durch das Ausschneiden verursachten Fehler, und zudem sind die unterseeischen Topographien mit wenigen Ausnahmen älteren Datums und deshalb (namentlich bei Flussdeltas) ohnehin nicht mehr genau.

Die Tiefen, aus welchen die Wasserproben erhoben werden, stimmen praktisch nie mit den Höhenlinien der Karte überein. Für die Berechnungen müssen aber die Schichtflächen der Probenahmetiefen bekannt sein. Wir haben sie durch eine lineare Interpolation aus den Quadratwurzeln der topographischen Schichtflächen erhalten.

\section{Berechnung des Stoffinhalts}

Als Basis dient die altbekannte Formel: Inhalt $=$ Volumen $\times$ Konzentration.

Es werden folgende vereinfachende Annahmen getroffen:

Die Konzentration des zu berechnenden gelösten Stoffes verändert sich zwischen den analysierten Tiefen linear.

Die Quadratwurzeln der Schichtflächen verändern sich zwischen den betreffenden Tiefen ebenfalls linear (analog einem Kegelstumpf).

Die Konzentrationsfunktion ist gemäss unseren Annahmen von der Tiefe linear, die Volumenfunktion quadratisch abhängig. Miteinander multipliziert ergeben beide Funktionen eine Funktion dritten Grades. Bis zu diesem Grad reicht die präzise Annäherung der Keplerschen Fassregel:

$$
I=\frac{h}{6}\left(y_{1}+4 y_{m}+y_{2}\right)
$$

wobei

I Stoffinhalt

$\mathrm{y}_{1} \quad$ Konzentration oben $\times$ Fläche oben

$\mathrm{y}_{2}$ Konzentration unten $\times$ Fläche unten

$\mathrm{y}_{\mathrm{m}}$ (Konz. oben + Konz. unten $) / 2 \times[(\sqrt{\text { Fläche oben }}+\sqrt{\text { Fläche unten }}) / 2]^{2}$

«oben»=obere Begrenzung der betreffenden Schicht, «unten»=untere Begrenzung.

Durch Einführen der Konzentration und Umformen erweitert sich Formel (1) zu

$$
I=\frac{h}{12}\left[\left(f_{o}+f_{w}\right)^{2}\left(c_{o}+c_{u}\right)+2 c_{o} f_{o}^{2}+2 c_{u} f_{u}^{2}\right]
$$


wobei

$f_{o}$ Quadratwurzel der Fläche oben

$c_{o} \quad$ Konzentration oben

$f_{u}$ Quadratwurzel der Fläche unten

$c_{u} \quad$ Konzentration unten

\section{Das Volumen berechnet sich als}

$$
V=\frac{h}{3}\left[f_{o}^{2}+f_{u}^{2}+\left(f_{o} \cdot f_{u}\right)\right]
$$

Beide Formeln (2) und (3) lassen sich leicht auf Rechnern programmieren, sogar auf Kleinrechnern. Sie sind in den Datenbekanntgabeprogrammen der Abteilung Limnologie der EAWAG [1] eingefügt und berechnen für jede Seeuntersuchung routinemässig neben anderen Summenwerten auch das (häufig sehr nützliche) Seetotal und das Seevolumen.

\section{RÉSUME}

Une formule pour la quantité totale des substances dissoutes dans un lac

Le calcul de la quantité totale est utilisé pour les bilans. Les superficies des différentes profondeurs sont découpées avec des ciseaux dans une carte topographique et pesées.

La formule est le produit de la fonction linéaire de la concentration selon la profondeur multiplié avec le volume d'un cône tronqué et donne le contenu dans une couche du lac.

Contenu d'une couche:

$$
\frac{\Delta z}{12}\left[\left(f_{s}+f_{i}\right)^{2} \cdot\left(c_{s}+c_{i}\right)+2 c_{s} f_{s}^{2}+2 c_{i} f_{1}\right]
$$

$f_{s} \quad$ Racine carrée de la superficie supérieure

$f_{i}$ Racine carrée de la superficie inférieure

$c_{s}$ Concentration à la profondeur supérieure

$c_{i}$ Concentration à la profondeur inférieure

$\Delta \mathbf{z}$ Epaisseur de la couche (différences des profondeurs)

La somme des contenues des couches fait le total.

\section{LITERATURVERZEICHNIS}

1 Bührer, H.: Computerprogramm zur Bekanntgabe aktueller Seedaten. Schweiz. Z. Hydrol. 37, 332-346 (1975).

Adresse des Autors: Dr. H. Bührer, Eidg. Anstalt für Wasserversorgung, Abwasserreinigung und Gewässerschutz (EAWAG), CH-8600 Dübendorf. 